\title{
Reconstruction with a Bovine Pericardial Patch of Infected Juxtarenal Aortic
} Aneurysm

\author{
Hideyuki Harada*, Xiaoning Tong, Naoya Kuriyama, Daita Kobayashi, Fumiaki Kimura and Toshio Baba \\ Department of Cardiovascular Surgery, Kushiro Kojinkai Memorial Hospital, Japan
}

*Corresponding author: Hideyuki Harada, Department of Cardiovascular Surgery, Kushiro Kojinkai Memorial Hospital, Japan, Tel: +81-0154-392533; Fax: +81-0154-54-0330; E-mail: h_harada@kojinkai.or.jp

Received: 13 Feb, 2019 | Accepted: 4 Mar, 2019 | Published: 8 Mar, 2019

Citation: Harada H, Tong X, Kuriyama N, Kobayashi D, Kimura F, et al. (2019) Reconstruction with a Bovine Pericardial Patch of Infected Juxtarenal Aortic Aneurysm. J Clin Case Stu 4(1): dx.doi.org/10.16966/2471-4925.184

Copyright: (C) 2019 Harada H, et al. This is an open-access article distributed under the terms of the Creative Commons Attribution License, which permits unrestricted use, distribution, and reproduction in any medium, provided the original author and source are credited.

\begin{abstract}
An infected aortic aneurysm (IAA) is a rare and fatal disease. Especially, it is challenging to repair IAA at the level of the renal artery. A 76-year-old male was referred to our hospital because of low grade fever, a flank pain, and lumbago for 1-month duration. The infected and juxtarenalsaccular formed aortic aneurysm was diagnosed by biochemistry examinations and computed tomography angiogram. We started intravenous antibiotic treatment. A patch repair of the aneurysmal neck using a bovine pericardial patch was performed under renal hypothermia. Long-term antibiotic therapy was given postoperatively. No signs of recurrent infection and aneurysmal dilatation of the aorta were observed for 5 years after surgery. Bovine pericardial patch plasty could be one of the surgical options for IAA, especially in patients with severe adhesion to the aneurysm.
\end{abstract}

Keywords: Infected aortic aneurysm; Juxtarenal aortic aneurysm; Bovine pericardial patch plasty

\section{Introduction}

An infected aortic aneurysm (IAA) is a rare and fatal disease. Surgical treatment for an IAA is challenging and the ideal surgical procedures and graft material remain subjects of discussion. Especially, an IAA at the level of the renal artery is hard to repair [1].

In view of the anatomic characteristic of saccular form in IAA, we completed a patch repair of the aortic intima defect using a bovine pericardial patch.

\section{Case Report}

A 76-year-old male was referred to our hospital because of low grade fever, a flank pain, and lumbago for 1-month duration. His past history involved type 2 diabetes and hypertension. Biochemistry examinations presented a white blood cell count of $8100 / \mathrm{mm}^{3}$ and a C-reactive protein (CRP) level of $3.88 \mathrm{mg} / \mathrm{dl}$. An immediate computed tomography angiogram was performed, which showed a saccular formed aneurysm and adjacent opacified extra-aortic space located at the postero-lateral wall of the abdominal aorta between the renal arteries (Figure 1). The infected and juxtarenal aortic aneurysm (JAA) was diagnosed. We started intravenous antibiotic treatment (Sulbactam/Ampicillin, $3 \mathrm{~g}$ daily for $8 \mathrm{~h}$ ). Seven days later, CRP level $(<0.10 \mathrm{mg} / \mathrm{dl})$ showed normal range. Blood culture was negative. The operation was performed using a midline abdominal incision (Figure 2). Severe adhesion around the aneurysm and the right renal artery was observed. The supraceliac and infrarenal abdominal aorta and the superior mesenteric and left renal arteries were exposed. After systemic heparinization, the supraceliac and infrarenal abdominal aorta and the superior mesenteric and left renal arteries were clamped and a vertical aortotomy was made at the level of the renal arteries by mobilizing the left renal vein in a caudad direction. A $3.5 \times 1.5 \mathrm{~cm}$ defect of the intima, from which the aneurysm had arisen, was found in the posterior part of the abdominal aorta. A 6-French gauge Foley balloon catheters were inserted in the orifice of the renal arteries from within the opened aorta. Renal hypothermia was provided with cold saline solution. Back-bleeding from the celiac artery was stopped using 5-French gauge Fogarty balloon catheter which was inserted from within the opened aorta. Debridement of infected tissues including infected arterial wall and numerous irrigation of the aneurysmal sac were performed, thereafter, the defect was repaired by using bovine pericardial patch plasty with 3-0 polyproplylene running sutures. After the aortotomy was closed using bovine pericardial strips, we performed omental wrapping (Figure 3). Renal artery perfusion time was 45 minutes. Culture from abdominal aneurysmal tissue was negative. The patient was discharged from hospital 17 days after surgery without the need for antibiotic therapy (Sulbactam/Ampicillin). Thereafter, oral antibiotic treatment (Levofloxacin) was continued for 11 months. The patient is alive and well 5 years after his operation (Figure 4).

\section{Discussion}

IAA is a complicated disease with difficult treatment and poor survival. In addition to systemic antibiotic therapy, removal of the infected aorta, debridement of the surrounding tissue, in situ aortic 


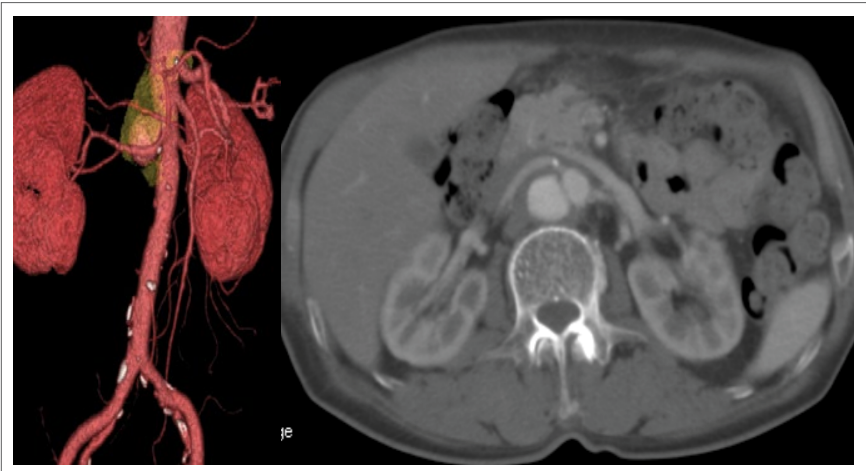

Figure 1: Preoperative abdominal CT angiography revealed a saccular formed aneurysm and adjacent opacified extra-aortic space (arrow), which were located at the postero-lateral wall of the abdominal aorta between the renal arteries.

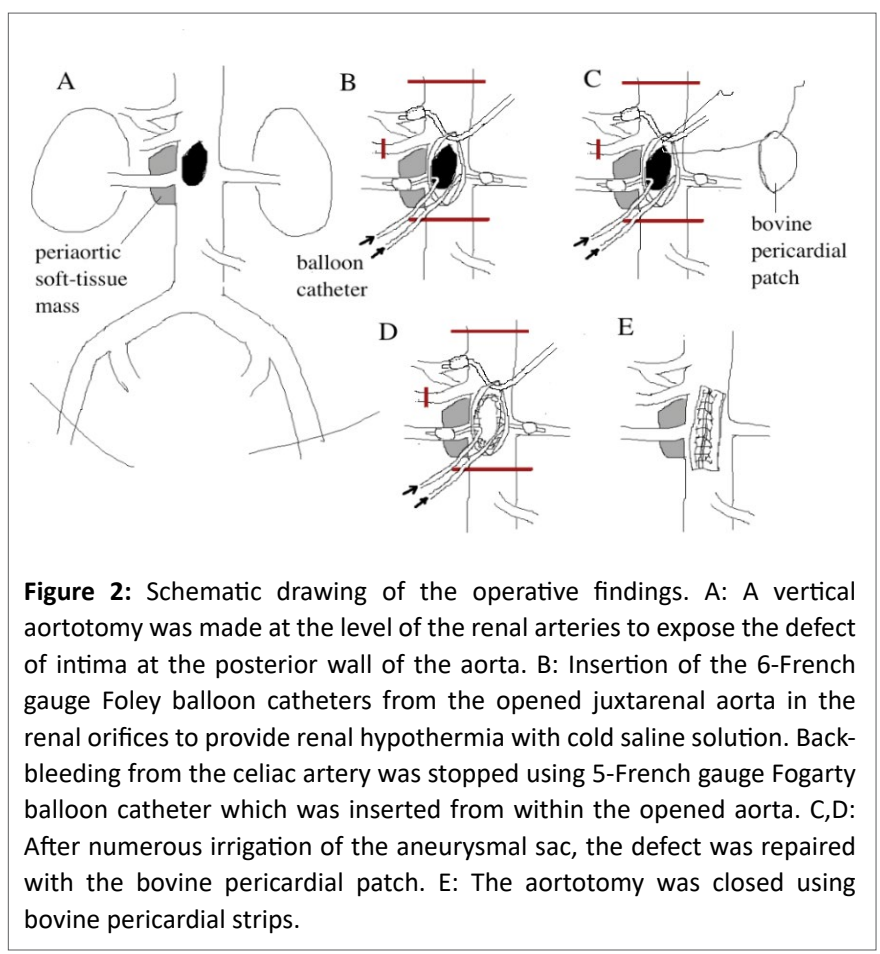

reconstruction, and omental wrapping is the accepted procedure for treating IAA, but the question of which graft material is optimal is still a matter of controversy.

A rifampicin-soaked Dacron graft [1], cryo preserved arterial allograft [2], superficial femoral vein [3], equine [4] or bovine pericardial roll graft [5], and great saphenous vein spiral graft [6] as surgical material have been reported. Although use of an autologous arterial patch for IAA has been reported [7], to our knowledge, there have been no cases except for the case who we have previously described [8] that underwent reconstructive surgery using bovine pericardial patch plasty. This procedure has low invasiveness because it minimizes the dissection range around the aneurysm, and handling of bovine pericardium is simple, which may shorten the operative time. In addition, bovine pericardium causes fewer cases of infection due to the antimicrobial effect of glutaraldehyde compared with those caused by a Dacron graft [9] which suggests that patch angioplasty using bovine pericardium is useful for treatment of saccular IAA that is difficult to dissect. However, when the tissue is fragile and patch

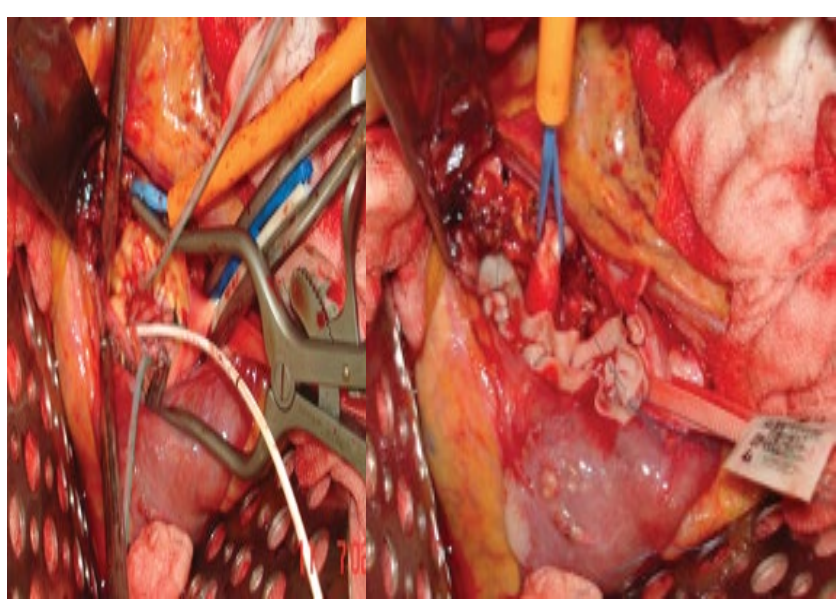

Figure 3: Operative findings. A: A 6-French gauge Foley balloon catheters were inserted in the orifice of the renal arteries from within the opened aorta. Renal hypothermia was provided. Back-bleeding from the celiac artery was stopped using 5-French Fogarty balloon catheter inserted from within the opened aorta. The defect was repaired with the bovine pericardial patch. B: The aortotomy was closed using bovine pericardial strips.

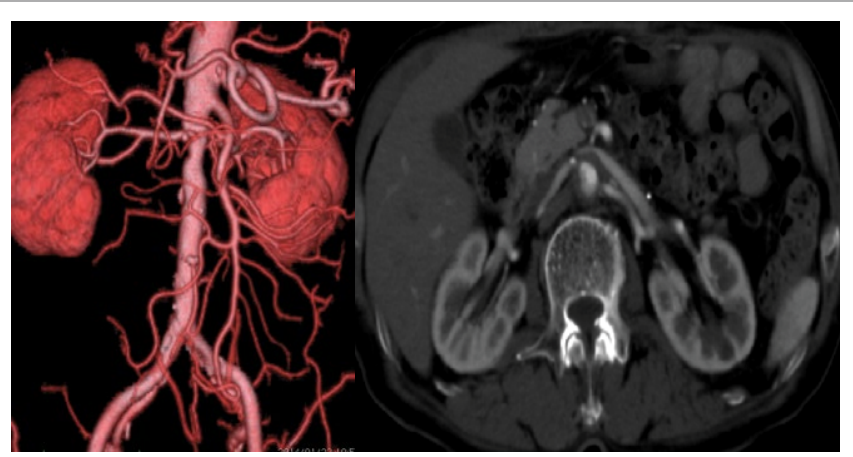

Figure 4: Postoperative abdominal CT angiography (20 months after surgery).

angioplasty is considered difficult, it is important to resect the fragile tissue and infection focus immediately and perform in situ aortic replacement. False aneurysm formation long after patch angioplasty of saccular aortic aneurysm with a synthetic graft has been reported [10], suggesting the necessity of careful follow-up.

Considering the characteristic feature of saccular formin IAA and to save the renal ischemic time, we completed a bovine pericardial patch repair of the aneurysmal neck. We found that the renal ischemic time was tolerable with this procedure. A standardized strategy to routinely apply renal hypothermia during the ischemic period of elective JAA surgery may protect renal function [11].

Endovascular aortic repair (EVAR) is a less invasive option to traditional open repair of IAA. A major demerit is that the infected tissue, including the aneurysm itself, is not removed, which may result in persistent sepsis and infection of endoprosthesis. EVAR could be considered a temporizing option or a bridge to later elective open repair [12].

Because long-term results of this procedure are still unknown, long-term antibiotic treatment was given postoperatively. 


\section{Conclusion}

We present a case of the successful surgical treatment for an IAA using a bovine pericardial patch. Because this technique is simple and less invasive than the standard procedure, it could be one of the surgical options for IAAs, especially in patients with severe adhesion to the aneurysm.

\section{References}

1. Kim YW (2010) Infected Aneurysm: Current Management. Ann Vasc Dis. 3: 7-15.

2. Brown KE, Heyer K, Rodriguez $\mathrm{H}$, Eskandari MK, Pearce WH, et al. (2009) Arterial reconstruction with cryopreserved human allografts in the setting of infection: A single-center experience with midterm follow-up. J Vasc Surg 49: 660-666.

3. Ohki S, Hirai H, Yasuhara K, Hatori K, Miki T, et al. (2016) Aortoiliac Artery Reconstruction Using Bilateral Reversed Superficial Femoral Veins for an Infected Abdominal Aortic Aneurysm. Ann Vasc Dis 9: 70-72.

4. Yamamoto H, Yamamoto F, Ishibashi K, Motokawa M (2009) In situ replacement with equine pericardial roll grafts for ruptured infected aneurysms of the abdominal aorta. J Vasc Surg 49: 1041-1045.

5. Czerny $M$, von Allmen $R$, Opfermann $P$, Sodeck $G$, Dick $F$, et al. (2011) Self-made pericardial tube graft: a new surgical concept for treatment of graft infections after thoracic and abdominal aortic procedures. Ann Thorac Surg 92: 1657-1662.
6. Aerts PD, van Zitteren M, Van Kasteren ME, Buiting AG, Heyligers JM, et al. (2013) Report of two in situ reconstructions with a saphenous spiral vein graft of Coxiella burnetii-infected aneurysms of the abdominal aorta. J Vasc Surg 57: 234-237.

7. Lee SK, Lee KB, Kim DI, Kim YW (2007) Autologous Arterial Patch Closure of a Mycotic, Inter-renal Aortic Aneurysm. EJVES Extra 14: 25-27.

8. Harada H, Tong X, Kuriyama N, Kimura F (2018) Bovine Pericardial Patch Plasty for Ruptured Infected Abdominal Aortic Aneurysm in the Aged. Japanese J Vasc Surg 27.

9. Kubota $H$, Endo $H$, Noma $M$, Ishii $H$, Tsuchiya $H$, et al. (2015) Xenopericardial roll graft replacement for infectious pseudoaneurysms and graft infections of the aorta. J Cardiothorac Surg 10: 133.

10. Okita Y, Takamoto S, Ando M, Morota T, Yamaki F, et al. (1997) Longterm results of patch repair for saccular aneurysms of the transverse aortic arch. Eur J Cardiothorac Surg 11: 953-956.

11. Yeung KK, Jongkind V, Coveliers HME, Tangelder GJ, Wisselink W (2008) Routine Continuous Cold Perfusion of the Kidneys during Elective Juxtarenal Aortic Aneurysm Repair. Eur J Vasc Endovasc Surg 35: 446-451.

12. Sörelius K, Mani K, Björck M, Sedivy P, Wahlgren CM, et al. (2014) Endovascular treatment of mycotic aortic aneurysms: a European multicenter study. Circulation 130: 2136-2142. 